\title{
Lorentz and $S U(3)$ Groups Derived from Cubic Quark Algebra
}

\author{
R. Kerner
}

Dedicated to Jiři Niederle on the occasion of his 70-th birthday

\begin{abstract}
We show how Lorentz and $S U(3)$ groups can be derived from the covariance principle conserving a $Z_{3}$-graded three-form on a $Z_{3}$-graded cubic algebra representing quarks endowed with non-standard commutation laws. This construction suggests that the geometry of space-time can be considered as a manifestation of symmetries of fundamental matter fields.
\end{abstract}

1. Many fundamental properties of matter at the quantum level can be announced without mentioning the space-time realm. The Pauli exclusion principle, symmetry between particles and anti-particles, electric charge and baryonic number conservation belong to this category. Quantum mechanics itself can be formulated without any mention of space, as was shown by M. Born, P. Jordan and W. Heisenberg [1] in their version of matrix mechanics, or in J. von Neumann's [2] formulation of quantum theory in terms of $C^{*}$ algebras. Non-commutative geometry [4] gives another example of interpreting space-time relationships in pure algebraic terms.

Einstein's dream was to be able to derive the properties of matter, and perhaps its very existence, from the singularities of fields defined on space-time, and if possible, from the geometry and topology of spacetime itself. A follower of Maxwell and Faraday, he believed in the primary role of fields and tried to derive the equations of motion as characteristic behavior of field singularities, or singularities of the space-time (see [3]).

One can defend an alternative point of view supposing that the existence of matter is primary with respect to that of space-time. In this light, the idea of deriving the geometric properties of space-time, and perhaps its very existence, from fundamental symmetries and interactions proper to matter's most fundamental building blocks seems quite natural.

If the space-time is to be derived from the interactions of fundamental constituents of matter, then it seems reasonable to choose the strongest ineractions available, which are the interactions between quarks. The difficulty resides in the fact that we should define these "quarks" (or their states) without any mention of space-time.

The minimal requirements for the definition of quarks at the initial stage of model building are the following:

i) The mathematical entities representing quarks should form a linear space over complex numbers, so that we could produce their linear combinations with complex coefficients. ii) They should also form an associative algebra, so that their multilinear combinations may be formed;

iii) There should exist two isomorphic algebras of this type corresponding to quarks and anti-quarks, and the conjugation transformation that maps one of these algebras onto another, $\mathcal{A} \rightarrow \overline{\mathcal{A}}$.

iv) The three quark (or three anti-quark) and the quark-anti-quark combinations should be distinguished in a certain way, for example, they should form a subalgebra in the algebra spanned by the generators.

With this in mind we can start to explore the algebraic properties of quarks that would lead to more general symmetries, that of space and time, appearing as a consequence of covariance requirements imposed on the discrete relations between the generators of the quark algebra.

2. At present, the most successful theoretical descriptions of fundamental interactions are based on the quark model, despite the fact that isolated quarks cannot be observed. The only experimentally accessible states are either three-quark or three-anti-quark combinations (fermions) or quark-anti-quark states (bosons). Whenever one has to do with a tri-linear combination of fields (or operators), one must investigate the behavior of such states under permutations.

Let us introduce $N$ generators spanning a linear space over complex numbers, satisfying the following relations which are a cubic generalization of anti-commutation in the ususal (binary) case (see e.g. $[5,6])$ :

$$
\theta^{A} \theta^{B} \theta^{C}=j \theta^{B} \theta^{C} \theta^{A}=j^{2} \theta^{C} \theta^{A} \theta^{B},
$$

with $j=e^{i \pi / 3}$, the primitive cubic root of 1 . We have $\bar{j}=j^{2}$ and $1+j+j^{2}=0$. We shall also introduce a similar set of conjugate generators, $\bar{\theta}^{\dot{A}}$, $\dot{A}, \dot{B}, \ldots=1,2, \ldots, N$, satisfying a similar condition with $j^{2}$ replacing $j$ :

$$
\bar{\theta}^{\dot{A}} \bar{\theta}^{\dot{B}} \bar{\theta}^{\dot{C}}=j^{2} \bar{\theta}^{\dot{B}} \bar{\theta}^{\dot{C}} \bar{\theta}^{\dot{A}}=j \bar{\theta}^{\dot{C}} \bar{\theta}^{\dot{A}} \bar{\theta}^{\dot{B}},
$$

Let us denote this algebra by $\mathcal{A}$. We shall endow this algebra with a natural $Z_{3}$ grading, considering the 
generators $\theta^{A}$ as grade 1 elements, and their conjugates $\bar{\theta}^{\dot{A}}$ being of grade 2 . The grades add up modulo 3 , so that the products $\theta^{A} \theta^{B}$ span a linear subspace of grade 2 , and the cubic products $\theta^{A} \theta^{B} \theta^{C}$ are of grade 0 . Similarly, all quadratic expressions in conjugate generators, $\bar{\theta}^{\dot{A}} \bar{\theta}^{\dot{B}}$ are of grade $2+2=4_{\bmod 3}=1$, whereas their cubic products are again of grade 0 , like the cubic products of $\theta^{A}$ 's.

Combined with the associativity, these cubic relations impose a finite dimension on the algebra generated by $Z_{3}$ graded generators. As a matter of fact, cubic expressions are the highest order that does not vanish identically. The proof is immediate:

$$
\begin{aligned}
\theta^{A} \theta^{B} \theta^{C} \theta^{D}= & j \theta^{B} \theta^{C} \theta^{A} \theta^{D}=j^{2} \theta^{B} \theta^{A} \theta^{D} \theta^{C}= \\
& j^{3} \theta^{A} \theta^{D} \theta^{B} \theta^{C}=j^{4} \theta^{A} \theta^{B} \theta^{C} \theta^{D}
\end{aligned}
$$

and because $j^{4}=j \neq 1$, the only solution is

$$
\theta^{A} \theta^{B} \theta^{C} \theta^{D}=0
$$

Therefore the total dimension of the algebra defined via the cubic relations (1) is equal to $N+N^{2}+\left(N^{3}-\right.$ $N) / 3$ : the $N$ generators of grade 1 , the $N^{2}$ independent products of two generators, and $\left(N^{3}-N\right) / 3$ independent cubic expressions, because the cube of any generator must be zero, and the remaining $N^{3}-N$ ternary products are divided by 3 , by virtue of the constitutive relations (1).

The conjugate generators $\bar{\theta}^{\dot{B}}$ span an algebra $\overline{\mathcal{A}}$ isomorphic with $\mathcal{A}$. Both algebras split quite naturally into sums of linear subspaces with definite grades:

$$
\mathcal{A}=\mathcal{A}_{0} \oplus \mathcal{A}_{1} \oplus \mathcal{A}_{2}, \quad \overline{\mathcal{A}}=\overline{\mathcal{A}}_{0} \oplus \overline{\mathcal{A}_{1}} \oplus \overline{\mathcal{A}}_{2},
$$

The subspaces $\mathcal{A}_{0}$ and $\overline{\mathcal{A}}_{0}$ form zero-graded subalgebras. These algebras can be made unital if we add to each of them the unit element $\mathbf{1}$ acting as identity and considered as being of grade 0 .

If we want the products between the generators $\theta^{A}$ and their conjugates $\bar{\theta}^{\dot{B}}$ to be included into the greater algebra spanned by both types of generators, we should consider all possible products, which will be included in the linear subspaces with a definite grade. of the resulting algebra $\mathcal{A} \otimes \overline{\mathcal{A}}$. In order to decide which expressions are linearly dependent, and what is the overall dimension of the enlarged algebra generated by $\theta^{A}$ 's and their conjugate variables $\bar{\theta}^{\dot{D}}$ 's, we must impose some binary commutation relations on their products.

The fact that conjugate generators are of grade 2 may suggest that they behave like products of two ordinary generators $\theta^{A} \theta^{B}$. Such a choice often was made (see, e.g., $[5,9,6]$ ). However, this does not enable one to make a distinction between conjugate generators and the products of two ordinary generators, and it would be better to be able to make the difference. Due to the binary nature of "mixed" products, another choice is possible, namely, to impose the following relations:

$$
\theta^{A} \bar{\theta}^{\dot{B}}=-j \bar{\theta}^{\dot{B}} \theta^{A}, \quad \bar{\theta}^{\dot{B}} \theta^{A}=-j^{2} \theta^{A} \bar{\theta}^{\dot{B}},
$$

In what follows, we shall deal with the first two simplest realizations of such algebras, spanned by two or three generators. Consider the case when $A, B, \ldots=$ 1,2 . The algebra $\mathcal{A}$ contains numbers, two generators of grade $1, \theta^{1}$ and $\theta^{2}$, their four independent products (of grade 2), and two independent cubic expressions, $\theta^{1} \theta^{2} \theta^{1}$ and $\theta^{2} \theta^{1} \theta^{2}$. Similar expressions can be produced with conjugate generators $\bar{\theta} \dot{C}$; finally, mixed expressions appear, like four independent grade 0 terms $\theta^{1} \bar{\theta}^{\dot{\mathrm{i}}}, \theta^{1} \bar{\theta}^{\dot{2}}, \theta^{2} \bar{\theta}^{\dot{\mathrm{i}}}$ and $\theta^{2} \bar{\theta}^{\dot{2}}$.

3. Let us consider multilinear forms defined on the algebra $\mathcal{A} \otimes \overline{\mathcal{A}}$. Because only cubic relations are imposed on products in $\mathcal{A}$ and in $\overline{\mathcal{A}}$, and the binary relations on the products of ordinary and conjugate elements, we shall fix our attention on tri-linear and bi-linear forms, conceived as mappings of $\mathcal{A} \otimes \overline{\mathcal{A}}$ into certain linear spaces over complex numbers.

Let us consider a tri-linear form $\rho_{A B C}^{\alpha}$. Obviously, as

$$
\rho_{A B C}^{\alpha} \theta^{A} \theta^{B} \theta^{C}=\rho_{B C A}^{\alpha} \theta^{B} \theta^{C} \theta^{A}=\rho_{C A B}^{\alpha} \theta^{C} \theta^{A} \theta^{B},
$$

by virtue of the commutation relations (1) it follows that we must have

$$
\rho_{A B C}^{\alpha}=j^{2} \rho_{B C A}^{\alpha}=j \rho_{C A B}^{\alpha} .
$$

Even in this minimal and discrete case, there are covariant and contravariant indices: the lower case and the upper case indices display inverse transformation properties. If a given cyclic permutation is represented by a multiplication by $j$ for the upper indices, the same permutation performed on the lower indices is represented by multiplication by the inverse, i.e. $j^{2}$, so that they compensate each other. Similar reasoning leads to the definition of the conjugate forms $\rho_{\dot{C} \dot{B} \dot{A}}^{\dot{\alpha}}$ satisfying the relations (5) with $j$ replaced by $j^{2}$ :

$$
\bar{\rho}_{\dot{A} \dot{B} \dot{C}}^{\dot{\alpha}}=j \bar{\rho}_{\dot{B} \dot{C} \dot{A}}^{\dot{\alpha}}=j^{2} \bar{\rho}_{\dot{C} \dot{A} \dot{B}}^{\dot{\alpha}}
$$

In the case of two generators, there are only two independent sets of indices. Therefore the upper indices $\alpha, \dot{\beta}$ take on the values 1 or 2 . We choose the following notation:

$$
\rho_{121}^{1}=j \rho_{112}^{1}=j^{2} \rho_{211}^{1} ; \quad \rho_{212}^{2}=j \rho_{221}^{2}=j^{2} \rho_{122}^{2},
$$

all other components identically vanishing. The conjugate matrices $\bar{\rho}_{\dot{B} \dot{C} \dot{A}}^{\dot{\alpha}}$ are defined by the same formulae, with $j$ replaced by $j^{2}$ and vice versa.

The constitutive cubic relations between the generators of the $Z_{3}$ graded algebra can be considered as intrinsic if they are conserved after linear transformations with commuting (pure number) coefficients, i.e. if they are independent of the choice of the basis. Let 
$U_{A}^{A^{\prime}}$ denote a non-singular $N \times N$ matrix, transforming the generators $\theta^{A}$ into another set of generators, $\theta^{B^{\prime}}=U_{B}^{B^{\prime}} \theta^{B}$. The primed indices run over the same range of values, i.e. from 1 to 2 ; the prime is there just to make clear we are referring to a new basis.

We are looking for the solution of the covariance condition for the $\rho$-matrices:

$$
\Lambda_{\beta}^{\alpha^{\prime}} \rho_{A B C}^{\beta}=U_{A}^{A^{\prime}} U_{B}^{B^{\prime}} U_{C}^{C^{\prime}} \rho_{A^{\prime} B^{\prime} C^{\prime}}^{\alpha^{\prime}}
$$

Let us write down the explicit expression, with fixed indices $(A B C)$ on the left-hand side. Let us choose one of the two available combinations of indices, $(A B C)=$ (121); then the upper index of the $\rho$-matrix is also fixed and equal to 1 :

$$
\Lambda_{1}^{\alpha^{\prime}} \rho_{121}^{1}=U_{1}^{A^{\prime}} U_{2}^{B^{\prime}} U_{1}^{C^{\prime}} \rho_{A^{\prime} B^{\prime} C^{\prime}}^{\alpha^{\prime}}
$$

Now, $\rho_{121}^{1}=1$, and we have two equations corresponding to the choice of values of the index $\alpha^{\prime}$ equal to 1 or 2. For $\alpha^{\prime}=1^{\prime}$ the $\rho$-matrix on the right-hand side is $\rho_{A^{\prime} B^{\prime} C^{\prime}}^{1^{\prime}}$, which has only three components,

$$
\rho_{1^{\prime} 2^{\prime} 1^{\prime}}^{1^{\prime}}=1, \quad \rho_{2^{\prime} 1^{\prime} 1^{\prime}}^{1^{\prime}}=j^{2}, \quad \rho_{1^{\prime} 1^{\prime} 2^{\prime}}^{1^{\prime}}=j,
$$

which leads to the following equation:

$$
\begin{aligned}
\Lambda_{1}^{1^{\prime}} & =U_{1}^{1^{\prime}} U_{2}^{2^{\prime}} U_{1}^{1^{\prime}}+j^{2} U_{1}^{2^{\prime}} U_{2}^{1^{\prime}} U_{1}^{1^{\prime}}+j U_{1}^{1^{\prime}} U_{2}^{1^{\prime}} U_{1}^{2^{\prime}}= \\
& =U_{1}^{1^{\prime}}\left(U_{2}^{2^{\prime}} U_{1}^{1^{\prime}}-U_{1}^{2^{\prime}} U_{2}^{1^{\prime}}\right)=U_{1}^{1^{\prime}}[\operatorname{det}(U)], \quad(10)
\end{aligned}
$$

because $j^{2}+j=-1$. For the alternative choice $\alpha^{\prime}=2^{\prime}$ the $\rho$-matrix on the right-hand side is $\rho_{A^{\prime} B^{\prime} C^{\prime}}^{2^{\prime}}$, whose three non-vanishing components are

$$
\rho_{2^{\prime} 1^{\prime} 2^{\prime}}^{2^{\prime}}=1, \quad \rho_{1^{\prime} 2^{\prime} 2^{\prime}}^{2^{\prime}}=j^{2}, \quad \rho_{2^{\prime} 2^{\prime} 1^{\prime}}^{2^{\prime}}=j .
$$

the corresponding equation gives:

$$
\Lambda_{1}^{2^{\prime}}=-U_{1}^{2^{\prime}}[\operatorname{det}(U)]
$$

The remaining two equations are obtained in a similar manner, resulting in the following:

$$
\Lambda_{2}^{1^{\prime}}=-U_{2}^{1^{\prime}}[\operatorname{det}(U)], \quad \Lambda_{2}^{2^{\prime}}=U_{2}^{2^{\prime}}[\operatorname{det}(U)] .
$$

The determinant of the $2 \times 2$ complex matrix $U_{B}^{A^{\prime}}$ appears everywhere on the right-hand side. Taking the determinant of the matrix $\Lambda_{\beta}^{\alpha^{\prime}}$ one gets immediately

$$
\operatorname{det}(\Lambda)=[\operatorname{det}(U)]^{3} .
$$

Taking into account that the inverse transformation should exist and have the same properties, we arrive at the conclusion that $\operatorname{det} \Lambda=1$,

$$
\operatorname{det}\left(\Lambda_{\beta}^{\alpha^{\prime}}\right)=\Lambda_{1}^{1^{\prime}} \Lambda_{2}^{2^{\prime}}-\Lambda_{1}^{2^{\prime}} \Lambda_{2}^{1^{\prime}}=1
$$

which defines the $S L(2, \mathbf{C})$ group, the covering group of the Lorentz group.

However, the $U$-matrices on the right-hand side are defined only up to the phase, which due to the cubic character of the relations (10-12), and they can take on three different values: $1, j$ or $j^{2}$, i.e. the matrices $j U_{B}^{A^{\prime}}$ or $j^{2} U_{B}^{A^{\prime}}$ satisfy the same relations as the matrices $U_{B}^{A^{\prime}}$ defined above. The determinant of $U$ can take on the values $1, j$ or $j^{2}$ while $\operatorname{det}(\Lambda)=1$

Let us then choose the matrices $\Lambda_{\beta}^{\alpha^{\prime}}$ to be the usual spinor representation of the $S L(2, \mathbf{C})$ group, while the matrices $U_{B}^{A^{\prime}}$ will be defined as follows:

$$
U_{1}^{1^{\prime}}=j \Lambda_{1}^{1^{\prime}}, U_{2}^{1^{\prime}}=-j \Lambda_{2}^{1^{\prime}}, U_{1}^{2^{\prime}}=-j \Lambda_{1}^{2^{\prime}}, U_{2}^{2^{\prime}}=j \Lambda_{2}^{2^{\prime}},
$$

the determinant of $U$ being equal to $j^{2}$.

Obviously, the same reasoning leads to the conjugate cubic representation of $S L(2, \mathbf{C})$ if we require the covariance of the conjugate tensor

$$
\bar{\rho}_{\dot{D} \dot{E} \dot{F}}^{\dot{\beta}}=j \bar{\rho}_{\dot{E} \dot{F} \dot{D}}^{\dot{\beta}}=j^{2} \bar{\rho}_{\dot{F} \dot{D} \dot{E}}^{\dot{\beta}}
$$

by imposing the equation similar to (8)

$$
\Lambda_{\dot{\beta}}^{\dot{\alpha}^{\prime}} \bar{\rho}_{\dot{A} \dot{B} \dot{C}}^{\dot{\beta}}=\bar{\rho}_{\dot{A}^{\prime} \dot{B}^{\prime} \dot{C}^{\prime}}^{\dot{\prime}^{\prime}} \bar{U}_{\dot{A}}^{\dot{A}^{\prime}} \bar{U}_{\dot{B}}^{\dot{B}^{\prime}} \bar{U}_{\dot{C}}^{\dot{C}^{\prime}}
$$

Matrix $\bar{U}$ is the complex conjugate of matrix $U$, and $\operatorname{det}(\bar{U})$ is equal to $j$.

Moreover, the two-component entities obtained as images of cubic combinations of quarks, $\psi^{\alpha}=$ $\rho_{A B C}^{\alpha} \theta^{A} \theta^{B} \theta^{C}$ and $\bar{\psi}^{\dot{\beta}}=\bar{\rho}_{\dot{D} \dot{E} \dot{F}}^{\dot{\beta}} \bar{\theta}^{\dot{D}} \bar{\theta}^{\dot{E}} \bar{\theta}^{\dot{F}}$ should anticommute, because their arguments do so, by virtue of (4):

$$
\left(\theta^{A} \theta^{B} \theta^{C}\right)\left(\bar{\theta}^{\dot{D}} \bar{\theta}^{\dot{E}} \bar{\theta}^{\dot{F}}\right)=-\left(\bar{\theta}^{\dot{D}} \bar{\theta}^{\dot{E}} \bar{\theta}^{\dot{F}}\right)\left(\theta^{A} \theta^{B} \theta^{C}\right)
$$

We have found the way to derive the covering group of the Lorentz group acting on spinors via the usual spinorial representation. The spinors are obtained as the homomorphic image of a tri-linear combination of three quarks (or anti-quarks). The quarks transform with matrices $U$ (or $\bar{U}$ for the anti-quarks), but these matrices are not unitary: their determinants are equal to $j^{2}$ or $j$, respectively. So, quarks cannot be put on the same footing as classical spinors; they transform under a $Z_{3}$-covering of the $S L(2, \mathbf{C})$ group.

A similar covariance requirement can be formulated with respect to the set of 2 -forms mapping the quadratic quark-anti-quark combinations into a fourdimensional linear real space. As we saw already, the symmetry (4) imposed on these expressions reduces their number to four. Let us define two quadratic forms, $\pi_{A \dot{B}}^{\mu}$ and conjugate $\bar{\pi}_{\dot{B} A}^{\mu}$ with the following symmetry requirement

$$
\pi_{A \dot{B}}^{\mu} \theta^{A} \bar{\theta}^{\dot{B}}=\bar{\pi}_{\dot{B} A}^{\mu} \bar{\theta}^{\dot{B}} \theta^{A}
$$

The Greek indices $\mu, \nu, \ldots$ take on four values, and we shall label them $0,1,2,3$. It follows immediately from (4) that

$$
\pi_{A \dot{B}}^{\mu}=-j^{2} \bar{\pi}_{\dot{B} A}^{\mu} .
$$


Such matrices are non-hermitian, and they can be realized by the following substitution:

$$
\pi_{A \dot{B}}^{\mu}=j^{2} i \sigma_{A \dot{B}}^{\mu}, \quad \bar{\pi}_{\dot{B} A}^{\mu}=-j i \sigma_{\dot{B} A}^{\mu}
$$

where $\sigma_{A \dot{B}}^{\mu}$ are the unit 2 matrix for $\mu=0$, and the three hermitian Pauli matrices for $\mu=1,2,3$.

Again, we want to get the same form of these four matrices in another basis. Knowing that the lower indices $A$ and $\dot{B}$ undergo the transformation with matrices $U_{B}^{A^{\prime}}$ and $\bar{U}_{\dot{B}}^{\dot{A}^{\prime}}$, we demand that there exist some $4 \times 4$ matrices $\Lambda_{\nu}^{\mu^{\prime}}$ representing the transformation of lower indices by the matrices $U$ and $\bar{U}$ :

$$
\Lambda_{\nu}^{\mu^{\prime}} \pi_{A \dot{B}}^{\nu}=U_{A}^{A^{\prime}} \bar{U}_{\dot{B}}^{\dot{B}^{\prime}} \pi_{A^{\prime} \dot{B}^{\prime}}^{\mu^{\prime}}
$$

and this defines the vector $(4 \times 4)$ representation of the Lorentz group. Introducing the invariant "spinorial metric" in two complex dimensions, $\varepsilon^{A B}$ and $\varepsilon^{\dot{A} \dot{B}}$ such that $\varepsilon^{12}=-\varepsilon^{21}=1$ and $\varepsilon^{\dot{1} \dot{2}}=-\varepsilon^{\dot{2} \dot{1}}$, we can define the contravariant components $\pi^{\nu A \dot{B}}$. It is easy to show that the Minkowskian space-time metric, invariant under the Lorentz transformations, can be defined as

$$
g^{\mu \nu}=\frac{1}{2}\left[\pi_{A \dot{B}}^{\mu} \pi^{\nu A \dot{B}}\right]=\operatorname{diag}(+,-,-,-)
$$

Together with the anti-commuting spinors $\psi^{\alpha}$ the four real coefficients defining a Lorentz vector, $x^{\mu}=$ $\pi_{A \dot{B}}^{\mu} \theta^{A} \bar{\theta}^{\dot{B}}$, can now generate the supersymmetry via standard definitions of super-derivations.

4. Consider now three generators, $Q^{a}, a=1,2,3$, and their conjugates $\bar{Q}^{\dot{b}}$ satisfying similar cubic commutation relations as in the two-dimensional case:

$$
\begin{gathered}
Q^{a} Q^{b} Q^{c}=j Q^{b} Q^{c} Q^{a}=j^{2} Q^{c} Q^{a} Q^{b}, \\
\bar{Q}^{\dot{a}} \bar{Q}^{\dot{b}} \bar{Q}^{\dot{c}}=j^{2} \bar{Q}^{\dot{b}} \bar{Q}^{\dot{c}} \bar{Q}^{\dot{a}}=j \bar{Q}^{\dot{c}} \bar{Q}^{\dot{a}} \bar{Q}^{\dot{b}}, \\
Q^{a} \bar{Q}^{\dot{b}}=-j \bar{Q}^{\dot{b}} Q^{a} .
\end{gathered}
$$

With the indices $a, b, c, \ldots$ ranging from 1 to 3 we get eight linearly independent combinations of three undotted indices, and the same number of combinations of dotted ones. They can be arranged as follows:

$$
\begin{gathered}
Q^{3} Q^{2} Q^{3}, \quad Q^{2} Q^{3} Q^{2}, \quad Q^{1} Q^{2} Q^{1}, \\
Q^{3} Q^{1} Q^{3}, \quad Q^{1} Q^{2} Q^{1}, \quad Q^{2} Q^{1} Q^{2}, \\
Q^{1} Q^{2} Q^{3}, \quad Q^{3} Q^{2} Q^{1},
\end{gathered}
$$

while the quadratic expressions of grade $0, Q^{a} \bar{Q}^{\dot{b}}$ span a 9-dimensional subspace in the finite algebra generaterd by $Q^{a}$ 's. The invariant 3 -form mapping these combinations onto some eight-dimensional space must also have eight independent components (over real numbers). The three-dimensional "cubic matrices" are then as follows:

$$
\begin{array}{lll}
K_{121}^{3+}=1, & K_{112}^{3+}=j^{2}, & K_{211}^{3+}=j ; \\
K_{212}^{3-}=1, & K_{221}^{3-}=j^{2}, & K_{122}^{3-}=j ; \\
K_{313}^{2+}=1, & K_{331}^{2+}=j^{2}, & K_{133}^{2+}=j ; \\
K_{131}^{2-}=1, & K_{113}^{2-}=j^{2}, & K_{311}^{2-}=j ; \\
K_{232}^{1+}=1, & K_{223}^{1+}=j^{2}, & K_{322}^{1+}=j ; \\
K_{323}^{1-}=1, & K_{332}^{1-}=j^{2}, & K_{233}^{1-}=j ; \\
K_{123}^{7}=1, & K_{231}^{7}=j^{2}, & K_{312}^{7}=j ; \\
K_{132}^{8}=1, & K_{321}^{8}=j^{2}, & K_{213}^{8}=j .
\end{array}
$$

all other components being identically zero.

Let the capital Greek indices $\Phi, \Omega$ take on the values from 1 to 8 . As in the case of the $\rho^{\alpha}$ matrices, we define the conjugate matrices $\bar{K}^{\dot{\Omega}}$, by replacing $j$ by $j^{2}$ and vice versa in the matrices $K^{\Omega}$.

The ternary multiplication table for eight cubic matrices $K$, with the same definition as for the $\rho$ matrices,

$$
\left\{K^{\Gamma}, K^{\Pi}, K^{\Lambda}\right\}_{a b c}=\sum_{d, e, f=1}^{3} K_{d a e}^{\Gamma} K_{e b f}^{\Pi} K_{f c d}^{\Lambda}
$$

The $Z_{3}$ graded ternary commutator can be defined as follows:

$$
\begin{aligned}
\left\{K^{\Gamma}, K^{\Pi}, K^{\Lambda}\right\}_{Z_{3}}= & \left\{K^{\Pi}, K^{\Lambda}, K^{\Gamma}\right\}+ \\
& j\left\{K^{\Pi}, K^{\Lambda}, K^{\Gamma}\right\}+ \\
& j^{2}\left\{K^{\Gamma}, K^{\Pi}, K^{\Lambda}\right\}
\end{aligned}
$$

The ternary multiplication table for these cubic matrices shall contain $8 \times 8 \times 8=512$ entries, and we cannot print it here due to the lack of place. Nevertheless, there are some interesting properties that can be noticed when one gets a closer look at the structure of the defining table above.

There are three distinct groups of two generators, each of them reproducing the structure of $\rho$-matrices, only with a different choice of two indices: $(1,2), 2,3$ nd 3,1 . They obviously reproduce the multiplication rules of the $\rho$-matrices. The last two generators are new in the sense that the combinations with three different indices did not exist in the previous twodimensional case. Their $Z_{3}$-graded ternary commutators vanish, which reproduces the behavior of two generators of the Cartan subalgebra of $S U(3)$.

There is one drawback here, namely, the multiplication does not close under the $Z_{3}$-graded ternary commutator: one needs to form real and imaginary combinations of $K$ and $\bar{K}$ cubic matrices in order to make the corresponding ternary algebra complete.

The covariance principle applied to the cubic matrices $K_{a b c}^{\Phi}$ underlinear change of the basis from $\theta^{a}$ to $\theta^{a^{\prime}}=U_{b}^{a^{\prime}} \theta^{b}$ means that we want to solve the following equations:

$$
S_{\Omega}^{\Phi^{\prime}} K_{d e f}^{\Omega}=K_{a^{\prime} b^{\prime} c^{\prime}}^{\Phi^{\prime}} U_{d}^{a^{\prime}} U_{e}^{b^{\prime}} U_{f}^{c^{\prime}}
$$


It takes more time to prove, but the result is that the $8 \times 8$ matrices $S_{\Omega}^{\Phi^{\prime}}$ are the adjoint representation of the $S U(3)$ group, whereas the $3 \times 3$ matrices $U_{d}^{a^{\prime}}$ are the fundamental representation of the same group, up to the phase factor that can take on the values $1, j$ or $j^{2}$.

The nine independent two-forms $P_{a \dot{b}}^{i}=-j^{2} \bar{P}_{\dot{b} a}^{i}$ transform as the $3 \otimes \overline{3}$ representation of $S U(3)$

Finally, the elements of the tensor product of both types of $j$-anti-commuting entities, $\theta^{A}$ and $Q^{b}$ can be formed, giving six quarks, $Q_{a}^{B}$, transforming via $Z_{3}$ coverings of $S L(2, \mathbf{C})$ and $S U(3)$, which looks very much like the three flavors.

5 . We have shown how the requirement of covariance of $Z_{3}$-graded cubic generalization of anticommutation relations leads to spinor and vector representations of the Lorentz group and the fundamental and adjoint representations of the $S U(3)$ group, thus giving the cubic $Z_{3}$-graded quark algebra the primary role in determining the Lorentz and $S U(3)$ symmetries. However, these representations coincide with the usual ones only when applied to special combinations of quark variables, cubic (spinor) or quadratic (vector) representations of the Lorentz group.

While acting on quark variables, the representations correspond to the $Z_{3}$-covering of groups. In this sense quarks are not like ordinary spinors or fermions, and as such, do not obey the usual Dirac equation. If the sigma-matrices are to be replaced by the nonhermitian matrices $\pi_{A \dot{B}}^{\mu}$, instead of the usual wave-like solutions of Dirac's equation we shall get the exponentials of complex wave vectors, and such solutions cannot propagate. Nevertheless, as argued in [9], certain tri-linear and bi-linear combinations of such solutions behave as usual plane waves, with real wave vectors and frequencies, if there is a convenient coupling of non-propagating solutions in the $k$-space.

\section{Acknowledgement}

We are greatly indebted to Michel Dubois-Violette for numerous discussions and enlightening remarks.

\section{References}

[1] Born, M., Jordan, P.: Zeitschrift fur Physik 34 858-878 (1925); ibid Heisenberg, W., 879-890 (1925).

[2] von Neumann, J.: Mathematical Foundations of Quantum Mechanics, Princeton Univ. Press (1996).

[3] Einstein, A., Infeld, L.: The Evolution of physics, Simon and Schuster, N.Y. (1967).

[4] Dubois-Violette, M., Kerner, R., Madore, J.: Journ. Math. Phys. 31, 316-322 (1990); ibid, 31, 323-331 (1990).

[5] Kerner, R.: Journ. Math. Phys., 33, 403-411 (1992).

[6] Abramov, V., Kerner, R., Le Roy, B.: Journ. Math. Phys., 38, 1650-1669 (1997).

[7] Lipatov, L. N., Rausch de Traubenberg, M., Volkov, G. G.: Journ. of Math. Phys. 49013502 (2008).

[8] Campoamor-Stursberg, R., Rausch de Traubenberg, M.: Journ. of Math. Phys. 49063506 (2008).

[9] Kerner, R.: Class. and Quantum Gravity, 14, A203-A225 (1997).

\section{Richard Kerner}

Laboratoire de Physique Théorique

de la Matière Condensée

Université Pierre-et-Marie-Curie - CNRS UMR 7600

Tour 22, 4-ème étage, Boite 121

4, Place Jussieu, 75005 Paris, France 